OPEN ACCESS

Edited by:

Maik Veste,

Brandenburg University of Technology Cottbus-Senftenberg, Germany

Reviewed by:

RongLiang Jia,

Northwest Institute of

Eco-Environment and Resources

(Chinese Academy of Sciences),

China

Giora Jehiel Kidron,

Hebrew University of Jerusalem, Israel

${ }^{*}$ Correspondence: Kirsten Fisher

kfisher2@calstatela.edu

Specialty section:

This article was submitted to Biogeography and Macroecology,

a section of the journal

Frontiers in Ecology and Evolution

Received: 07 October 2019 Accepted: 20 December 2019

Published: 21 January 2020

Citation:

Fisher K, Jefferson JS and Vaishampayan P (2020) Bacterial Communities of Mojave Desert Biological Soil Crusts Are Shaped by Dominant Photoautotrophs and the Presence of Hypolithic Niches.

Front. Ecol. Evol. 7:518

doi: 10.3389/fevo.2019.00518

\section{Bacterial Communities of Mojave Desert Biological Soil Crusts Are Shaped by Dominant Photoautotrophs and the Presence of Hypolithic Niches}

\author{
Kirsten Fisher $^{1 *}$, Jameka S. Jefferson ${ }^{1}$ and Parag Vaishampayan ${ }^{2}$ \\ ${ }^{1}$ Department of Biological Sciences, California State University, Los Angeles, CA, United States, ${ }^{2}$ Biotechnology and \\ Planetary Protection Group, Jet Propulsion Laboratory, California Institute of Technology, Pasadena, CA, United States
}

Biocrust communities are often heterogeneous and affected by small-scale environmental features, including both physical and biotic factors. The presence of moss in biocrusts, for example, is likely to create a set of physical conditions distinct from those found in microbial biocrusts that lack mosses. Similarly, hypolithic organisms living under and on the belowground surface of translucent and opaque stones (typically quartz) experience a distinct environment relative to surrounding soil surface organisms. To understand the impact of these factors on biocrust bacterial community composition, we performed 16S rRNA sequencing from surface and hypolithic biocrusts with and without the common biocrust moss, Syntrichia caninervis. While alpha diversity indices did not differ significantly between any of the biocrust habitats sampled, we did observe differences in overall community composition. Cyanobacterial abundance and diversity decreased in the presence of moss and in surface samples compared to hypolithic biocrusts, while Proteobacteria showed the opposite pattern. Acidobacteria were significantly more abundant in hypolithic niches, and Patescibacteria were found to be restricted to moss-dominated surface biocrusts. Notably, bacterial community composition was found to shift significantly between surface and hypolithic microbial biocrusts (Adonis, $R^{2}=0.122, p=0.002$ ) and between surface moss and microbial biocrusts $\left(R^{2}=0.107, p=0.002\right)$. These findings support the idea that even at small spatial scales (e.g., within $<10 \mathrm{~cm}$ ), desert biocrust bacterial community composition varies based on the habitat structure and cohabitants.

Keywords: biological soil crust, bacterial community, hypolithic, Syntrichia caninervis, 16S rRNA

\section{INTRODUCTION}

Competitive exclusion and habitat selection theory posit that differential habitat selection and niche partitioning may permit organisms of similar phenotypes to coexist (Darwin, 1859; Gause, 1934; Hutchinson, 1959; Rosenzweig, 1981). In soil microbial communities, diversity may be maintained through the spatiotemporal partitioning of habitats and associated resources (Ettema and Wardle, 2002; Lee et al., 2016). As a driver of community diversity, niche partitioning is an important 
process to understand in the context of global change, as higher diversity is expected to enhance the resilience of communities in the presence of disturbance (MacArthur, 1955; Giller et al., 1997; Girvan et al., 2005).

The importance of niche partitioning coupled with facilitative interactions is particularly pronounced for soil biological crust (biocrust) communities, which comprise diverse heterotrophic microorganisms that are dependent on the resources generated by photoautotrophs (Baran et al., 2015; Maier et al., 2018; Couradeau et al., 2019). Biocrust composition varies with physical factors as well as age and successional stage, but these communities may include fungi, lichens, mosses, green algae, diatoms, and archaea in addition to diverse bacteria (Belnap and Lange, 2003). Biocrusts predominate in dryland ecosystems, where they represent an estimated $40-50 \%$ of surface cover (Elbert et al., 2012; Garcia-Pichel et al., 2013) and are responsible for significant ecosystem services, including carbon cycling and nitrogen fixation (Belnap and Lange, 2003; Elbert et al., 2012).

Biocrusts are frequently considered successional communities, and successional stages are classified by the dominant photoautotroph, with early-stage biocrusts characterized by colonizing cyanobacteria (e.g., Microcoleus vaginatus) and mid to later stages dominated by lichens and mosses, respectively (Weber et al., 2016; Mogul et al., 2017; Maier et al., 2018). However, independent of succession, the presence or absence of particular biocrust photoautotrophs and their bacterial cohabitants can also be controlled by abiotic features of the local environment, such as moisture availability and substrate stability (Kidron et al., 2000, 2009). At meso- to micro-scales, the development of biocrusts and the establishment of their constituent photoautotrophs is dependent on edaphic factors (e.g., soil $\mathrm{pH}$, nutrients, and texture) and local habitat features such as slope, orientation, and shading from vascular plants (Bowker et al., 2016; Mogul et al., 2017; Durham et al., 2018). At this small local scale, biocrust organisms can be further partitioned into hypolithic habitats, which occur under the ventral surfaces of translucent and opaque stones (typically quartz) that are embedded in the soil surface (Chan et al., 2012). Hypolithic habitats are commonly found in drylands, although they can occur anywhere suitable substrate is available (Pointing, 2016). In addition to reduced UV radiation and buffering from extreme thermal fluctuations, organisms in these niche habitats experience enhanced water availability relative to that in surrounding surface soils due lower evaporation, higher relative humidity, and capture of water via fog condensation (Pointing, 2016). Nonetheless, dryland hypolithic habitats are still colonized by poikilohydric organisms that must withstand extended periods without water (Pointing and Belnap, 2012).

Although not always explicitly recognized as biocrusts, the microbial communities associated with hypolithic habitats have been well-characterized globally (Chan et al., 2012; Makhalanyane et al., 2013; Stomeo et al., 2013; Le et al., 2016; Vikram et al., 2016; Lacap-Bugler et al., 2017; Van Goethem et al., 2017). Cyanobacteria are the most common and abundant organisms in hypolithic biocrust communities (Chan et al., 2012; Pointing, 2016), with taxa from the genus Phormidium dominant in Antarctic and Tibetan hypoliths and Chroococcidiopsis most abundant in hot and cold deserts (Lacap-Bugler et al., 2017). Comparisons of microbial community composition in hypolithic and adjacent surface soil habitats have typically supported the idea that hypolithic niches harbor unique assemblages of bacteria that are distinct from, and not a filtered subset of, surface soil communities (Khan et al., 2011; Stomeo et al., 2013; but see also Makhalanyane et al., 2013; Le et al., 2016).

Although less common, hypolithic biocrusts sometimes include mosses (Cockell and Stokes, 2006; Cowan et al., 2010, 2011; Chan et al., 2012; de los Ríos et al., 2014). In surface biocrusts, the presence of mosses is assumed to modulate microbial community composition (Moquin et al., 2012; Antoninka et al., 2015; Xiao and Veste, 2017), presumably because their additional biomass modifies the physical and chemical environment experienced by the biocrust microbial community (Antoninka et al., 2015; Xiao et al., 2016). Mossdominated surface biocrusts are also capable of buffering important ecosystem functions such as nitrogen, carbon, and phosphorous cycling from the detrimental effects of increased aridity in deserts and drylands (Delgado-Baquerizo et al., 2016). Most work characterizing moss bacterial community composition has compared moss biocrusts to nearby soils that do not support biocrusts (Moquin et al., 2012; Delgado-Baquerizo et al., 2016; Xiao and Veste, 2017). However, studies directly comparing biocrusts with different dominant photoautotroph have found that moss-dominated biocrusts harbor a microbial community taxonomically and functionally distinct from that of cyanobacteria-dominated biocrusts (Kidron et al., 2010; Maier et al., 2018). Similarly, comparisons of bacterial community composition from hypolithic and adjacent surface niches thus far have focused on extreme environments (e.g., the Antarctic, Namib, and Atacama Deserts) that do not support welldeveloped surface biocrusts (Makhalanyane et al., 2013; Stomeo et al., 2013; Le et al., 2016; Vikram et al., 2016). Thus, the role that hypolithic niches interspersed within broader surface biocrusts might play in promoting local-scale beta diversity remains relatively unexplored.

Because of the potential contributions of habitat partitioning and facilitative interactions to the overall diversity and associated ecological resilience of biocrust microbial communities, in this study we set out to characterize the relative impacts of hypolithic niche and dominant photoautotroph on bacterial community composition. We sampled biocrusts with either cyanobacteria or moss as the dominant photoautotroph from both surface and hypolithic microhabitats within a small (8 $\mathrm{m}^{2}$ ) area in the Mojave Desert, and used 16s rDNA sequencing to test the hypothesis that both the presence of moss and hypolithic microhabitat would have significant impacts on the composition of bacterial communities associated with each type of biocrust.

\section{METHODS}

\section{Field Site and Sample Collection}

Soil and biocrust samples were collected on March 25, 2018 from the Sheep Creek Wash near Wrightwood, CA. The Sheep Creek Wash site is located at the northern base of the San Gabriel 
Mountains $\left(34^{\circ} 22^{\prime} 33.85^{\prime \prime} \mathrm{N}, 117^{\circ} 36^{\prime} 34,59 \mathrm{~W}\right)$ and the western edge of the Mojave Desert at an elevation of 1,800 m (Baughman et al., 2017). This site was chosen based on the presence of both surface and hypolithic Syntrichia caninervis biocrusts within the same restricted $\left(\sim 8 \mathrm{~m}^{2}\right)$ area. Syntrichia caninervis was detected based on characteristics such as hair points on the apices of leaves, leaf morphology, and colony pigmentation. Biocrusts with cyanobacteria as the dominant photoautotroph were identified based on their adherent texture and darker pigmentation relative to the underlying sand substrate. To differentiate the description of crust type based on dominant photoautotroph (cyanobacteriadominated vs. moss-dominated) from the description of biocrust bacterial community composition (which will likely always include cyanobacteria in some proportion), for the remainder of this report we use the term "microbial biocrust" to refer to biocrusts with cyanobacteria as the dominant photoautotroph. Seven replicate samples were collected for each of the following microsite types: surface moss biocrust; surface microbial biocrust; hypolithic moss biocrust; hypolithic microbial biocrust; sub-biocrust soil (for all previous sample types); and nonbiocrust surface soil. Abiotic features of the habitat such as slope, aspect, and sun exposure can influence the establishment of dominant photoautotrophs such as mosses and cyanobacteria (Kidron et al., 2000, 2009, 2010), and thus might also directly select for particular bacterial assemblages. Because we aimed to focus our study on the impact of biocrust mosses and hypolithic microsites on bacterial community composition, we designed our collection strategy to minimize variation in local abiotic factors across the biocrusts sampled. All samples were collected from the same shelf within the Sheep Creek Wash, which was $\sim 2 \times 4 \mathrm{~m}$ and contained a mosaic of intermixed microbial (cyanobacteria-dominated) and moss biocrusts with quartz and other stones sporadically embedded within. The sampling area experienced uniform shading from two trees (Juniperus occidentalis) to the east and southeast of the shelf. While there was some variation in the microtopography across the sampling area (1-3\% slope change from the center of the shelf to the lower edge), both surface and hypolithic moss and microbial biocrusts were distributed throughout the entire area, and replicate samples of each crust type were collected from both the interior and outer edges of the sampling area to capture the range of slope variation. For each hypolithic sample we collected, we also collected a sample of the surface biocrust (moss or microbial) directly abutting the hypolith quartz rock (Figure 1). To limit our comparisons to moss and microbial photoautotrophs, we avoided collecting biocrusts with lichens present. A sterile spatula (surface sterilized with $70 \%$ isopropyl alcohol between samples) was used for collection of 5-10 g soils and biocrusts, and each sample was placed individually into a sterile Nasco Whirl Pak bag (Fort Atkinson, WI). For hypolithic samples, quartz rocks (often with visible adhered microbial biomass) were collected along with soil and biocrust. All samples were stored on ice during field collection and transport back to the lab, where they were stored at $-20^{\circ} \mathrm{C}$ until DNA extraction.

\section{Sample Preparation and DNA Extraction}

Quartz samples were crushed with a UV sterilized hammer to obtain biological matter adhered to the rock samples. Smaller rocks were scraped using a sterile scalpel to gather biological materials for DNA extraction. For samples containing moss biocrusts, ca 5 stems of moss were first submerged for several seconds (using sterile forceps) in the buffer used during cell disruption step of the DNA extraction protocol, to remove some of the adhered soil and biocrust material for subsequent DNA extractions. Care was taken to remove all traces of moss after submersion. The prepared samples then underwent DNA extraction using a QIAGEN DNeasy PowerSoil Kit (Qiagen, Germantown, MD) according to the manufacturer's instructions (with the addition of the mosswashing step noted above). Quantification readings were taken immediately after DNA extraction using a Qubit 4 fluorometer (Invitrogen, Carlsbad, CA).

\section{6s rRNA Amplification and Sequencing}

The V3 and V4 hypervariable region within the $16 \mathrm{~S}$ rRNA cistron was amplified using primer sequences $341 \mathrm{~F}$ and $805 \mathrm{R}$ with Illumina sequencing adaptors (Herlemann et al., 2011; MizrahiMan et al., 2013). All PCR reactions contained $12.5 \mu \mathrm{l}$ of Apex HotStart Master Mix, $8.5 \mu$ l of sterilized molecular biology-grade water, $1.0 \mu \mathrm{l}$ of $10 \mathrm{mM}$ forward and reverse primers, and $2.0 \mu \mathrm{l}$ of DNA template. Each PCR reaction was performed using the following cycling parameters: initial denaturation of $95^{\circ} \mathrm{C}$ for $3 \mathrm{~min}$, denaturation of $95^{\circ} \mathrm{C}$ for $30 \mathrm{~s}$, annealing (generating 25 cycles) of $55^{\circ} \mathrm{C}$ for $30 \mathrm{~s}$, extension of $72^{\circ} \mathrm{C}$ for $30 \mathrm{~s}$, final extension of $72^{\circ} \mathrm{C}$ for $30 \mathrm{~s}$. Positive $(1 \mathrm{mg} / \mu \mathrm{l}$ bacterial community DNA, ZymoBIOMICS Microbial Community DNA Standard, Zymo Inc.) and negative controls were included in these PCR runs.

To visually confirm successful amplification, all PCR products were run in $0.5 \mathrm{X}$ TBE buffer on a $2 \%$ agarose gels. Each gel contained $6 \mu \mathrm{l}$ DNA Ladder II $1(1 \mathrm{~kb})$ and $6 \mu \mathrm{l}$ of each DNA sample, followed by staining with ethidium bromide. Upon confirmation of the target $\sim 500 \mathrm{bp}$ amplicons, PCR products were sent to ChunLab (ChunLab, Inc., Seoul, South Korea) for sequencing on the Illumina MiSeq platform. Because the negative controls failed to yield any amplification products, only positive controls were sent for sequencing.

\section{Sequence Assembly and Analysis}

Demultiplexed paired end MiSeq reads for 40 samples were trimmed, filtered, denoised, and merged using the DADA2 (Callahan et al., 2016) plugin in QIIME2 (Bolyen et al., 2018). Sequences were assigned to taxa using the scikit-learn 0.20.2 native Bayes classifier (Pedregosa et al., 2011) trained on the Silva v.132 reference database (Quast et al., 2013; Yilmaz et al., 2014). The q2-taxa plugin in QIIME2 was used to filter mitochondrial and chloroplast sequences from the dataset prior to downstream analyses.

For the calculation of phylogenetic diversity metrics (Faith's, unweighted UniFrac), positive control samples were removed and a rooted phylogeny was generated from the sequences using 


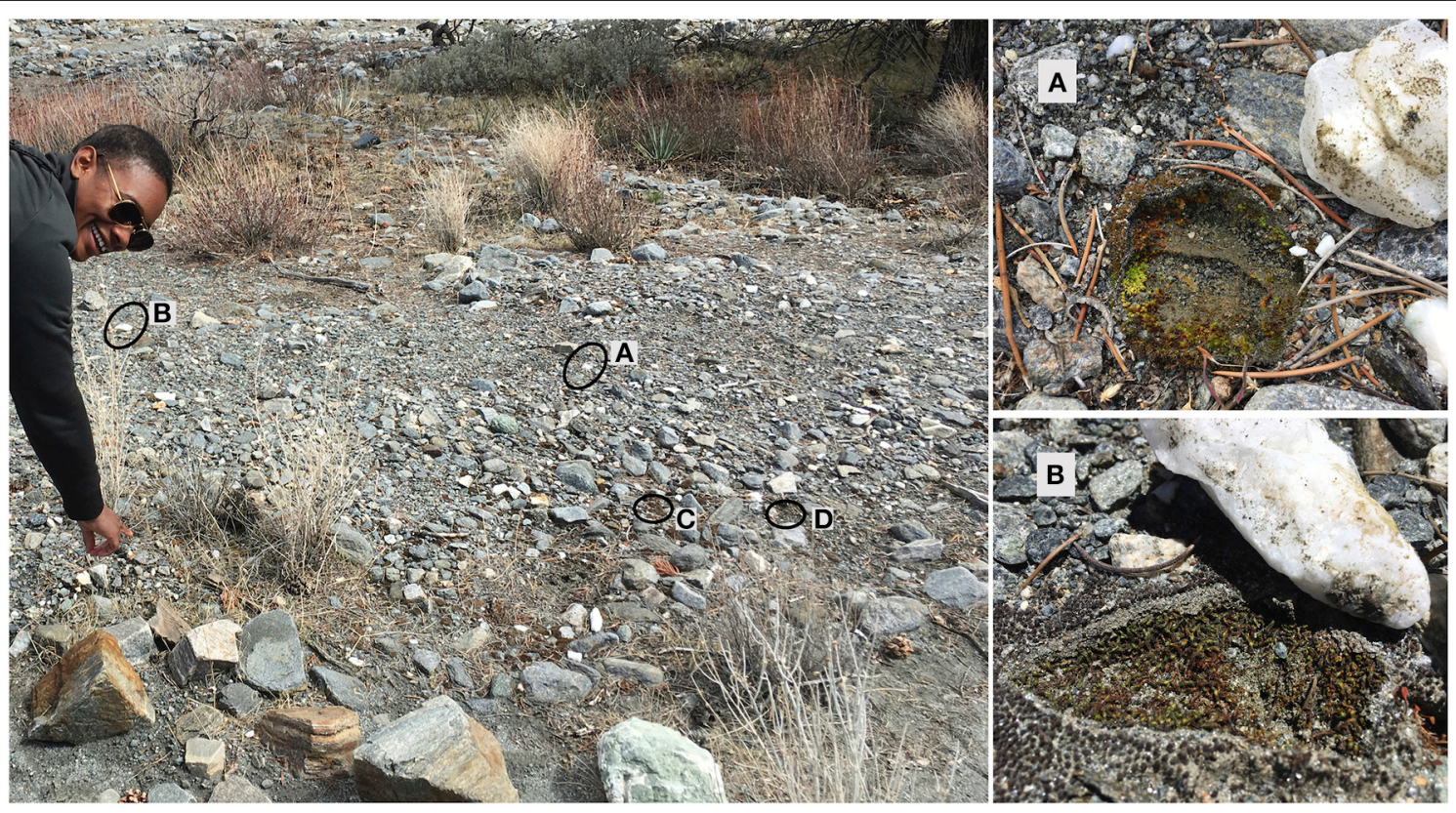

FIGURE 1 | Habitat photo (Left), showing a major portion of the collection area with locations of two hypoliths harboring moss biocrusts indicated $[\mathbf{A}, \mathbf{B}$ - corresponding to the photos in $(\mathbf{A}, \mathbf{B})$ to the right of the figure]. Representative moss (C) and microbial (D) biocrusts are also indicated. Inset $(\mathbf{A})$ : moss hypolith showing mosses in their hydrated state; the surface biocrust adjacent to this hypolith is microbial, toward the upper left of the panel. Inset (B): moss hypolith in desiccated state, with moss biocrusts on the soil surface adjacent to the hypolith.

the QIIME2 q2-phylogeny plugin's align-to-tree-mafft-fasttree pipeline. Standard alpha (Shannon's, evenness, Faith's) and beta (Bray-Curtis, unweighted and weighted UniFrac) diversity metrics were calculated with the QIIME2 q2-diversity plugin using a data set rarefied to 9,000 OTUs per sample. This sampling depth was selected based on rarefaction curves for observed OTUs generated for each sample type at sampling depths ranging from 1 to 10,000. Alpha diversity measures were explored for associations with habitat metadata (crust location and biocrust composition) to test for significant differences in community diversity between different biocrust microhabitats.

Sample metadata, the QIIME2 feature abundance table (containing the frequencies of each OTU), and the QIIME2 taxonomy table (containing the taxonomic assignments of each OTU) were imported into the Calypso online software suite for microbiome analysis (Zakrzewski et al., 2017). Prior to analyses, the two positive control samples and the non-crust (soil only) samples were excluded from the data set. Rare OTUs with $<0.01 \%$ relative abundance across all samples were also removed. Abundance data for the 3,418 taxa present in the remaining 28 samples were normalized through cumulative-sum scaling and $\log _{2}$ transformed.

An Adonis PERMANOVA of Bray-Curtis distances was used to test for significant compositional differences between moss and microbial biocrusts in surface and hypolithic niches. To compare overall community composition across biocrust sample types, we also performed non-metric multidimensional scaling using Bray-Curtis distances. Multivariate ordinations were performed first for all four biocrust sample types combined.
To better resolve the individual influences moss and hypolithic niche on community composition, we then performed Adonis and NMDS for pairwise comparisons of samples grouped by biocrust composition (with or without moss) and by location (surface or hypolithic). Univariate analyses (one-way ANOVA) were implemented to determine which taxa (phyla, genera) displayed significant differential abundance between sample types.

To generate taxonomic community profiles scaled to relative abundance within sample types, the original unfiltered, nonnormalized data were imported into phyloseq (McMurdie and Holmes, 2013), where the same filters were applied as described above. Samples from identical biocrust types were then merged and OTU abundance data were normalized to relative abundance within sample type prior to generating taxonomic community profiles.

\section{RESULTS}

\section{Sequence Assembly}

After filtering, denoising, merging, and removal of chimeric sequences, sequencing depth of the field-collected samples ranged from 4,184 to 20,010 (mean $=11,054)$.

The two mock microbial community positive control samples were consistent with each other in composition and did not share any sequences in common with the rest of the samples. Together they contained 22 OTUs, all of which represented sequences highly similar or identical to those from the eight species present in the ZymoBIOMICS standard. In total, 18,490 features were 
recovered in the assembly, with the depth of coverage per feature ranging from 1 to 2,284 $($ mean $=23)$.

\section{Diversity Measures}

Shannon indices did not indicate overall significant differences in alpha diversity among any of the sampled biocrust or soil habitats (Kruskal-Wallis $p=0.245$ ), and no differences in Shannon diversity were found in pairwise comparisons of samples filtered by biocrust composition (moss vs. microbial crust samples in hypolithic sites, $p=0.223$; in surface sites, $p=0.631$ ) or by sampling location (hypolithic vs. surface moss crusts, $p=0.584$; hypolithic vs. surface microbial crusts, $p=0.199$ ). Similar to Shannon diversity measures, Faith's Phylogenetic Diversity (PD) did not vary significantly in any of these pairwise comparisons. Evenness was not found to vary significantly among sample types overall (group Kruskal-Wallis $p=0.311$ ), although a pairwise comparison of microbial crusts did indicate significantly higher evenness of bacterial communities in hypolithic compared to surface sites $(p=0.045)$.

\section{Multivariate Analyses of Community Composition}

Adonis PERMANOVA of Bray-Curtis distances indicated significant overall differences in composition between biocrust sample types $\left(R^{2}=0.142, p=0.0003\right)$. Further partitioning of the sample data to pairwise comparisons within one biocrust type or one biocrust niche revealed these patterns to be driven largely by compositional differences between surface moss and microbial biocrusts $\left(R^{2}=0.092, p=0.038\right)$ and between surface and hypolithic biocrusts without moss $\left(R^{2}=0.115, p=0.002\right)$. Adonis did not distinguish samples from surface and hypolithic moss biocrusts $\left(R^{2}=0.88, p=0.08\right)$, or hypolithic moss and microbial biocrusts $\left(R^{2}=0.83, p=0.19\right)$.

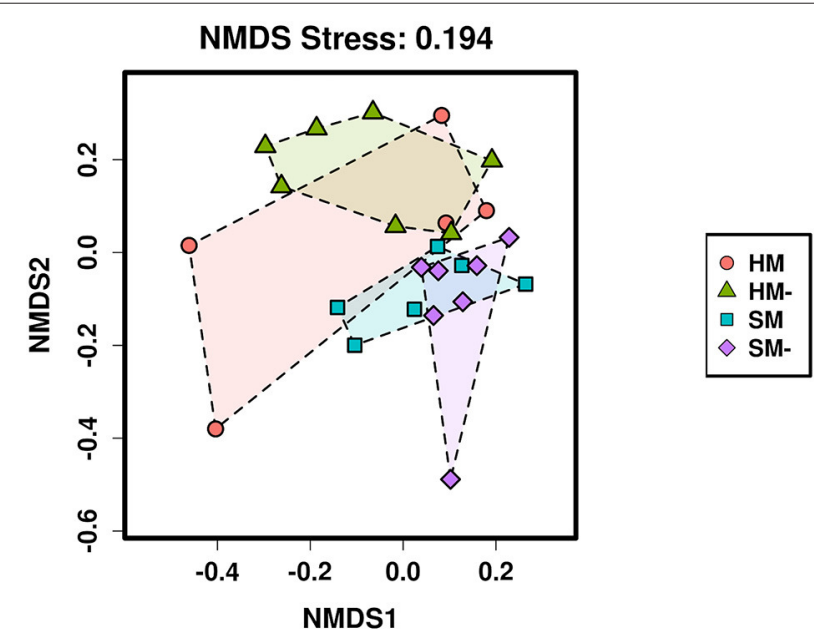

FIGURE 2 | Plot of the non-metric multidimensional scaling ordination of Bray-Curtis distances for the four sampled biocrust habitats. HM, hypolithic moss biocrust; HM-, hypolithic microbial biocrust; SM, surface moss biocrust; SM-, surface microbial biocrust.
Non-metric multidimensional scaling (NMDS) suggested compositional differences between at least some of the four biocrust habitats we sampled (Figure 2). When the samples were partitioned to compare composition within each type of biocrust by habitat and vice-versa, NMDS ordinations were consistent with the Adonis results, visually distinguishing communities from hypolithic and surface biocrusts without moss and from moss and microbial surface biocrusts (Figure 3).

\section{Community Profiles and Differential Taxon Abundance in Sampled Biocrusts}

Community profiles reflected shifts in the relative abundance of all phyla present in the sampled biocrust habitats, showing a trend toward increased cyanobacterial abundance in biocrusts without moss present (Figure 4). Relative abundance profiles of cyanobacterial genera across the four biocrust communities and adjacent non-biocrust soils revealed increased diversity of cyanobacterial taxa in hypolithic biocrusts compared to surface biocrusts and bare soils (Figure 5). ANOVA comparisons of phylum abundance indicated significant differences in the abundance of Chloroflexi ( $p=0.00056)$, Deinococcus-Thermus $(p=0.0024)$, Acidobacteria (0.015), and Elusimicrobia $(p=$ 0.021 ) within the 28 biocrust-associated samples (Figure 6). When samples were restricted to one sampling location (surface or hypolithic), surface environments $(n=16)$ showed significant differential abundance of Patescibacteria $(p=0.032)$, Chloroflexi $(p=0.038)$, and Planctomycetes $(p=0.042)$, all of which were more abundant in moss-dominated surface biocrusts. In hypolithic environments $(n=16)$, Elusimicrobia $(p=0.022)$, and Deinococcus-Thermus ( $p=0.04$ ) were differentially abundant with significantly higher prevalence in biocrusts without moss. Grouping samples by dominant photoautotroph (moss or microbial) in the ANOVA revealed that moss-dominated biocrusts $(n=16)$ harbored significantly more Acidobacteria $(p$ $=0.015)$ in hypolithic niches, while Planctomycetes $(p=0.021)$ and Patescibacteria $(p=0.027)$, were more abundant in surface moss biocrusts. In microbial biocrusts $(n=16)$, DeinococcusThermus ( $p=0.00073)$, Chloroflexi $(p=0.00087)$, Bacteriodetes $(p=0.34)$, and Acidobacteria $(p=0.41)$ all showed significant differential abundance, with all but Bacteriodetes having higher abundance in hypolithic compared to surface crusts lacking moss.

To provide more resolution regarding the taxa associated with significant shifts in community composition, ANOVA tests for differential abundance were run at the generic level for both surface moss and microbial biocrusts, and for microbial biocrusts in surface and hypolithic niches. In surface biocrusts, the presence of moss significantly increased the abundance of Tepidisphaera, Hymenobacter, Rhizobacter, Sphingomonas, Spriosoma, Bryobacter, and Pseudonocardia relative to biocrusts lacking moss, which supported significantly more Aquabacterium, Oligoflexus, Caenimonas, Sporocytophaga, Rhodocytophaga, and Arctibacter (Figure 7). In microbial biocrusts, hypolithic samples contained a significantly higher abundance of Truepera, Trichocoleus-SAG-26.92, Solirubrobacter, Rubrobacter, PMMR1, Phormidium-SAG-37.90, Parviterribacter, Flavitalea, 


\section{Microbial Biocrusts}

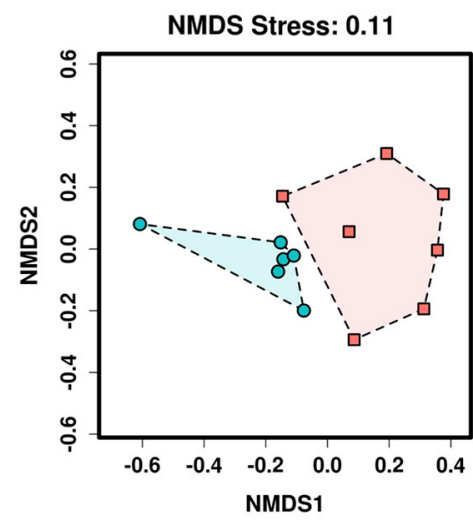

Hypolithic Biocrusts

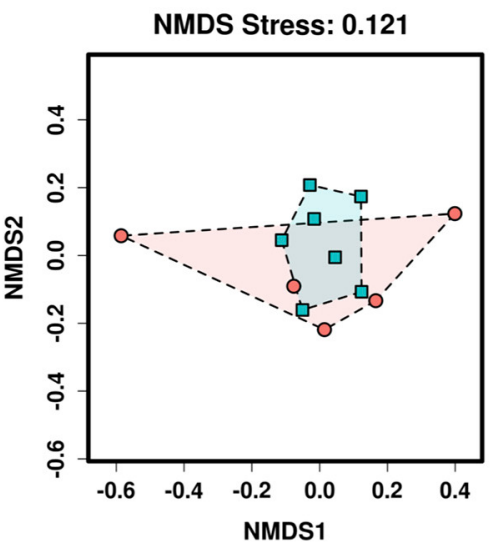

Moss Biocrusts

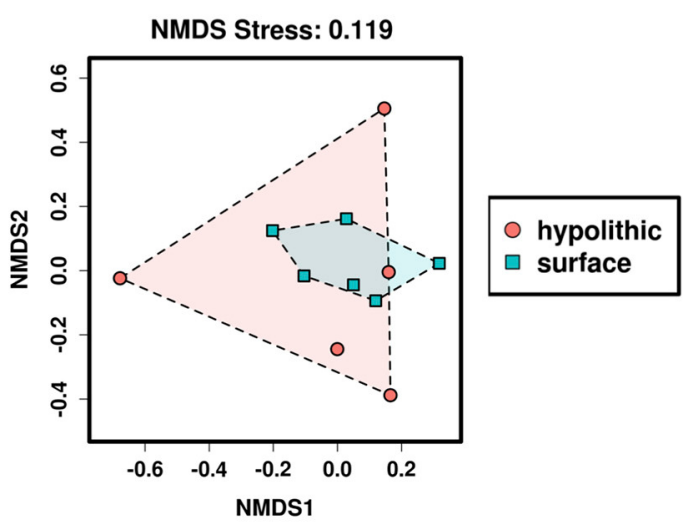

Surface Biocrusts

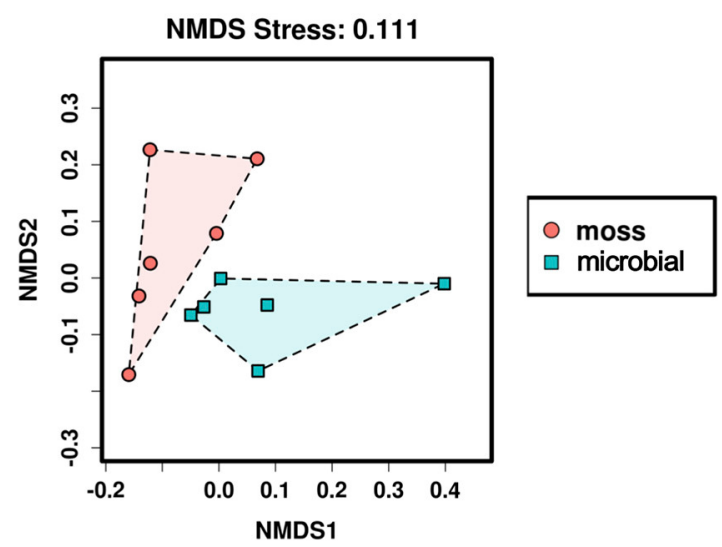

FIGURE 3 | Plots for pairwise non-metric multidimensional scaling (NMDS) ordinations of Bray-Curtis distances. Upper panels show comparisons of microbial (Left) and moss (Right) biocrusts from hypolithic and surface niches; lower panels show comparisons of microbial and moss biocrusts from hypolithic (Left) and surface (Right) niches.

Fimbriiglobus, Conexibacter, Bryobacter, and Angustibacter (Figure 8). Surface microbial biocrusts, in contrast, contained significantly higher abundance of Taibaiella, Sporocytophaga, Silvanigrella, Segetibacter, Pseudoxanthomonas, Peredibacter, Flavobacterium, Flavisolibacter, Fibrella, Cellvibrio, Blastococcus, and Aquabacterium (Figure 8).

\section{DISCUSSION}

Biocrusts and the essential ecosystem services that they provide are threatened by anthropogenic disturbances, including physical damage (Weber et al., 2016; Durham et al., 2018) and climate change (Belnap et al., 2004; Delgado-Baquerizo et al., 2016). Since biocrust resistance and resilience to such disturbances is likely to be influenced by community composition (Girvan et al., 2005), understanding factors that may impact local biocrust microbial diversity is critical for predicting responses to disturbance and designing effective restoration strategies.

The results of this study corroborate previous work noting the influence of dominant photoautotrophs in shaping bacterial community composition in surface biocrusts (Maier et al., 2014, 2018), as well as studies that have found compositional shifts with biocrust development or succession (Mogul et al., 2017; Maier et al., 2018). Consistent with these studies, we found significant differences in the composition of bacterial communities from surface moss- and cyanobacteria-dominated biocrusts (Maier et al., 2018). Contrary to these studies, we did not find significant differences in alpha diversity measures among the four biocrust community types we sampled (Maier et al., 2018). However, our study does suggest that beta diversity increases with the co-occurrence of biocrusts with and without moss as the dominant photoautotroph. 


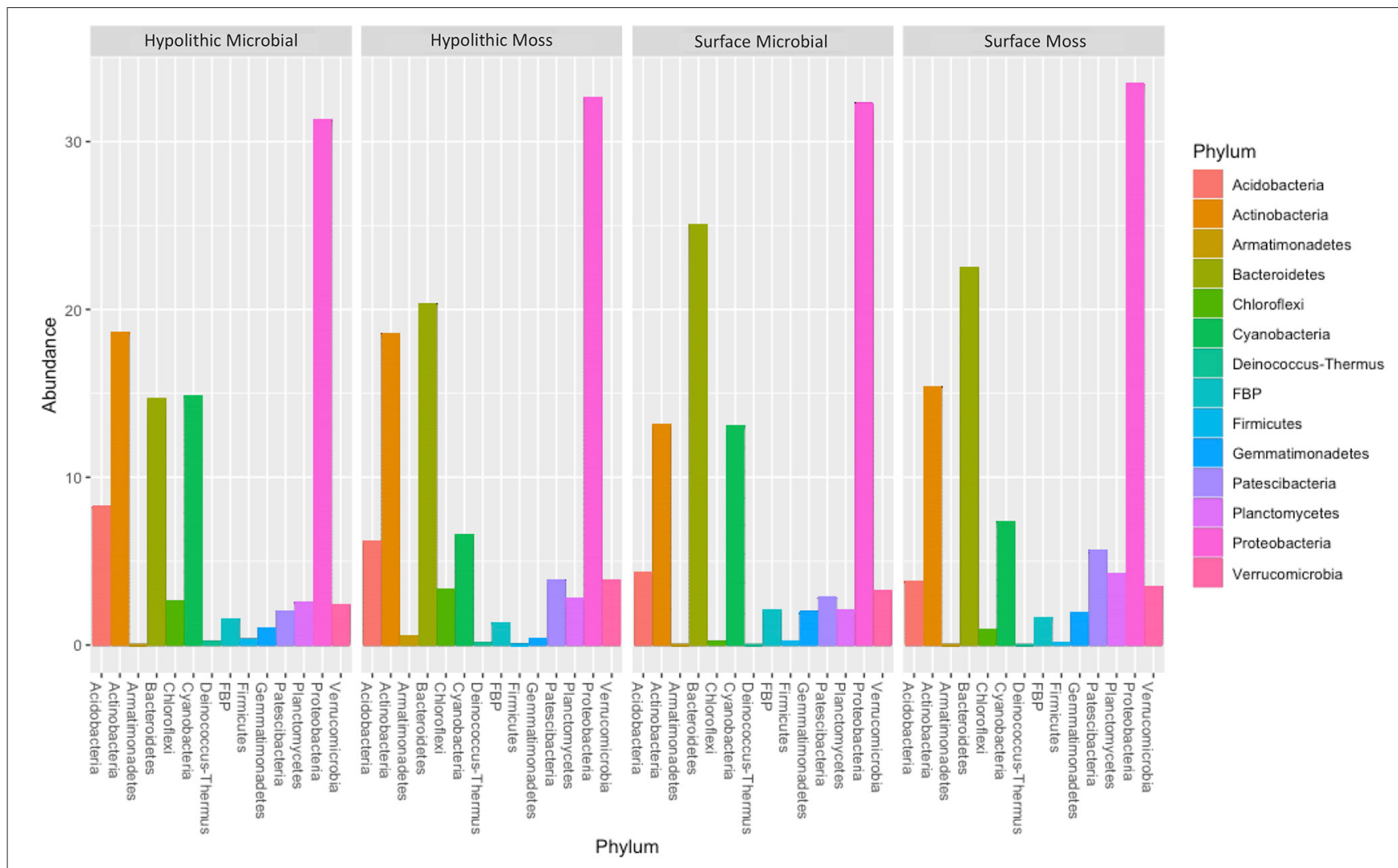

FIGURE 4 | Community profile plots showing the relative abundance of all phyla found in the four sampled biocrust habitats ( $N=7$ for each habitat). OTUs with $<0.01 \%$ abundance across all samples were removed prior to analysis; remaining abundance data were normalized through cumulative-sum scaling and log 2 transformed.

Unlike surface biocrusts, the communities associated with biocrusts in hypolithic spaces were not significantly impacted by the presence of moss, although we did observe an overall reduction in the abundance of cyanobacteria in hypoliths with moss. These results are consistent with the idea that hypolithic niches exert a strong selective habitat filter, as has been noted in comparisons of cyanobacterial community composition in hypolithic habitats from deserts worldwide (Lacap-Bugler et al., 2017). The unique selective filter imposed by the conditions in hypolithic spaces may attenuate species interactions such as competition, which could explain our observation that OTUs in hypolithic microbial biocrusts displayed significantly higher evenness compared to their surface counterparts.

Comparative work on hypolith bacterial community composition at a regional scale has focused hyperarid deserts that support very little soil biomass beyond hypolithic communities (Cowan et al., 2010; Stomeo et al., 2013; de los Ríos et al., 2014). Thus, comparisons of hypolith and non-hypolith communities in these studies have involved nearby soils without biocrusts, and have concluded that the community in hypolithic environments is primarily filtered from the regional pool of soil microbes (Makhalanyane et al., 2013). The processes influencing hypolith community composition are likely to be more dynamic in regions where both hypoliths and surface

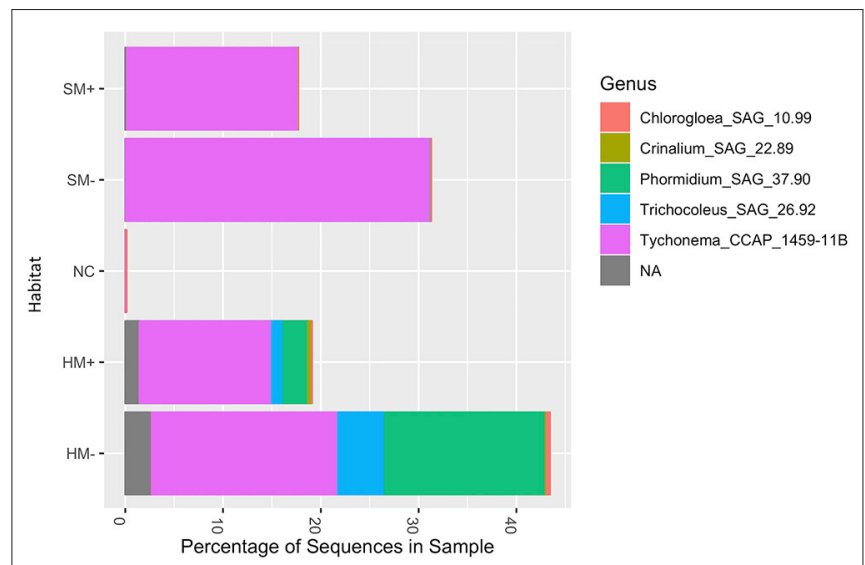

FIGURE 5 | Community profile plots showing the relative abundance of Cyanobacteria genera found the four sampled biocrust habitats and bare soil. OTUs with $<0.01 \%$ abundance across all samples were removed prior to analysis; remaining abundance data were normalized through cumulative-sum scaling and $\log _{2}$ transformed. HM, hypolithic moss biocrust; HM-, hypolithic microbial biocrust; SM, surface moss biocrust; SM-, surface microbial biocrust; NC, non-crusted bare soil.

biocrusts coexist, and in these systems, hypolithic communities may serve to "seed" surrounding soils with biocrust-forming taxa (Chan et al., 2012). 
The comparisons reported here provide some evidence supporting this refugial role for hypoliths. Two cyanobacterial taxa were significantly more abundant in hypolithic as opposed to surface microbial biocrusts, Phormidium SAG 37.90 ( $p=$ $0.0064)$ and Trichocoleus SAG $26.92(p=0.0036)$, which are associated with temperate freshwater (Marquardt and Palinska, 2007) and hot desert biocrust environments (Lange et al., 1992, as Microcoleus sociatus), respectively. These seemingly conflicting habitat associations may reflect the natural disturbance regime in the Sheep Creek Wash where we collected our samples: while the sampling area was located on an elevated, stable shelf within

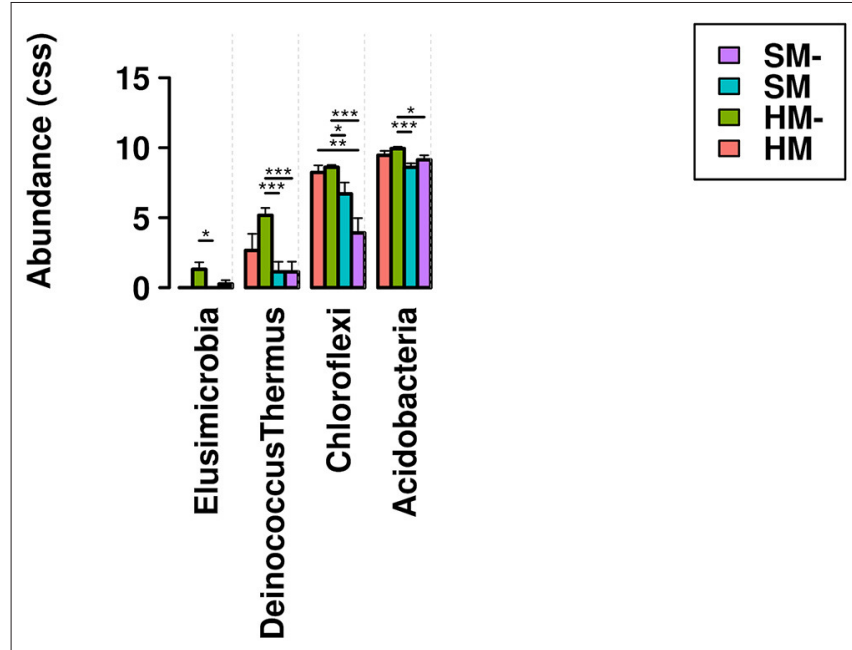

FIGURE 6 | Relative abundance of phyla found to be differentially abundant in an ANOVA of the four sampled biocrust habitats. Bars indicate significant pairwise differences at ${ }^{\star} \geq 0.05 ;{ }^{* \star} \geq 0.01 ;{ }^{\star \star *} \geq 0.001$. HM, hypolithic moss biocrust; HM-, hypolithic microbial biocrust; SM, surface moss biocrust; SM-, surface microbial biocrust. the shallow wash, it is likely that the seasonal flow of water in the wash occasionally covers the shelf. This periodic seasonal disturbance may explain the freshwater habitat associations for many of the taxa highlighted in our differential abundance analyses and community profiles. For example, both moss and microbial biocrusts in surface environments were dominated by a single genus of cyanobacteria, Tychonema CCAP 145911, an oscillatoroid species typically associated with freshwater environments (Suda et al., 2002). Surface biocrusts without moss are the least physically stable of the four biocrust types we sampled, and thus may be the most vulnerable to occasional disturbance. While periodic flooding may eliminate some taxa from biocrusts on the surface, these taxa may persist in protected hypolithic niches, or in more stable moss biocrusts (see below).

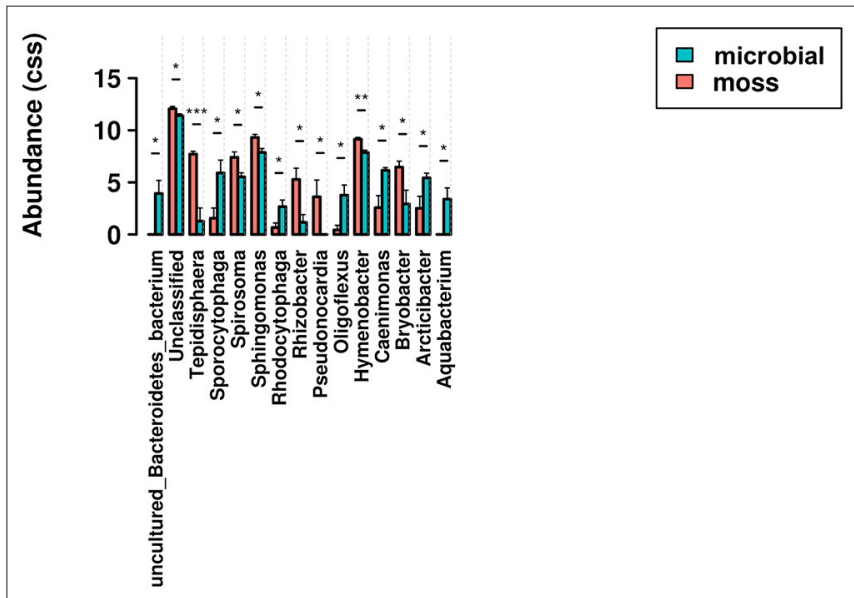

FIGURE 7 | Relative abundance of genera found to be differentially abundant in an ANOVA comparison of surface biocrusts with and without moss. Bars indicate significant pairwise differences at ${ }^{\star} \geq 0.05 ;{ }^{\star \star} \geq 0.01 ;{ }^{\star \star \star} \geq 0.001$.

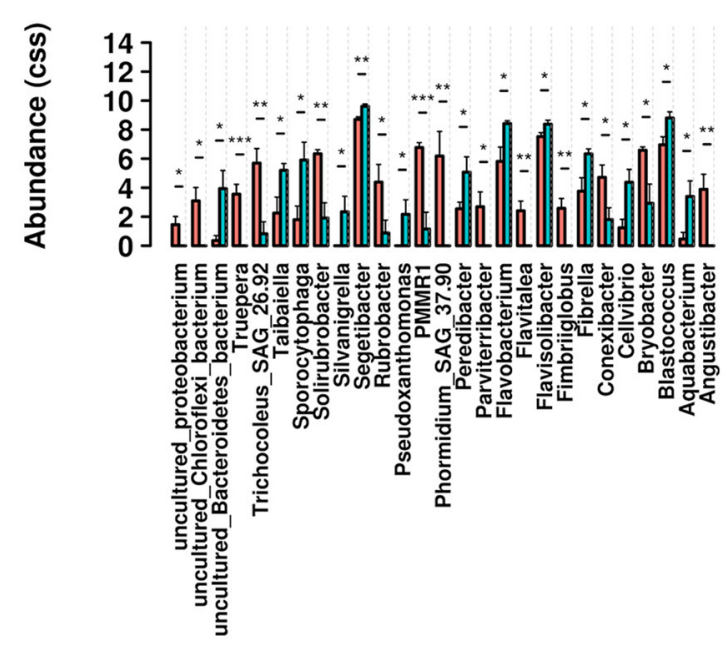


The two taxa for which we found the most significant increase in abundance from surface to hypolithic microbial biocrusts are both potential extremophiles, Truepera $(p=0.0005)$ and Caulobacteraceae sp. PMMR1 $(p=0.0004)$. This unexpected association of extremophilic taxa with hypolithic niches, where conditions are expected to be less stressful than on the surface, may reveal a role for hypolithic spaces in providing refugia from occasional disturbances.

In contrast to hypoliths without moss, hypolithic moss biocrust communities were not statistically different from surface moss biocrusts in the Adonis analysis or NMDS ordination. Similar to hypolithic niches, moss biocrusts on the surface may offer some physical protection from low-level disturbances like those described above. This result may also indicate that the presence of moss alters the physical environment in surface biocrusts such that light and moisture conditions experienced by microbes associated with moss biocrusts are similar enough to conditions in hypolithic niches to support partially overlapping bacterial communities.

Recent work on biocrust restoration has emphasized the utility of mosses such as Syntrichia caninervis and $S$. ruralis for reestablishing biocrust communities and their ecological function (Antoninka et al., 2015, 2018). Mosses are particularly promising components of biocrust restoration inocula, as they stabilize soils, enhance moisture retention, and facilitate the establishment of other functionally important biocrust microorganisms (Antoninka et al., 2015). Our results corroborate the role of mosses in modulating biocrust bacterial community composition, and expanded metagenomic investigation of the biocrusts investigated here will provide further resolution regarding the potential facilitative role of mosses by characterizing functional variation accompanying shifts in bacterial community composition with dominant photoautotroph. While the composition of communities in hypolithic spaces has previously been considered without direct comparison to nearby surface biocrusts, our results highlight the potential role of hypolithic niches in enhancing bacterial diversity at sites containing established surface biocrusts. If future biocrust restoration projects deploy greenhouse-grown biocrust mosses in an effort to expedite re-establishment of more complex, late-successional communities (Antoninka et al., 2018), such efforts might be enhanced by simultaneous introduction of quartz stones, sporadically embedded in the soil surface within the area under restoration (Chan et al., 2012). Deliberate introduction of such hypolithic spaces could encourage the establishment of taxa that might otherwise be absent, and could potentially provide refugia for later colonization of surface biocrusts once mosses become established.

By investigating the influence of both hypolithic niche and the presence of moss, our study highlights the possible overlapping roles of mosses and hypoliths in shaping biocrust bacterial community composition. The results presented here corroborate the influence of dominant photoautotrophs, like moss, on community composition (Maier et al., 2018). Overall, community composition is heavily impacted by hypolithic niche: teasing apart communities associated with one biocrust type (moss or microbial) within niche (surface or hypolith) allowed us to differentiate the potential effects of these factors. This approach revealed that hypolithic spaces appear to harbor a unique bacterial community that is relatively insensitive to the presence of moss. At our study site, this hypolithic community is distinct from that in surface biocrusts that do not include moss; however, the effect of hypolithic niche is diminished in comparisons of moss biocrusts from hypolithic and surface habitats. If moss biocrusts and hypoliths are partially redundant in their bacterial community composition due to similarities in the physical microhabitats that they create, this may have implications for the design and implementation of projects aiming to restore biocrusts in the presence of historical or ongoing disturbance.

\section{DATA AVAILABILITY STATEMENT}

The datasets generated for this study can be found in NCBI SRA, NCBI Accession No. PRJNA577884.

\section{AUTHOR CONTRIBUTIONS}

KF, JJ, and PV contributed to the conceptual development and design of the study, and wrote sections of the manuscript. JJ collected samples, completed all lab work prior to sequencing, and wrote the first draft of the manuscript. KF performed assembly and analyses. All authors contributed to manuscript revision, read and approved the submitted version.

\section{FUNDING}

Part of the research was carried out at the Jet Propulsion Laboratory, California Institute of Technology, under a contract with the National Aeronautics and Space Administration. This study was also supported in part by NSF DEB-1638996 to KF. Funds for open access publication are provided by the Cal State LA Library Open Access Author Fund.

\section{REFERENCES}

Antoninka, A. J., Bowker, M. A., Chuckran, P., Barger, N. N., Reed, S., and Belnap, J. (2018). Maximizing establishment and survivorship of field-collected and greenhouse-cultivated biocrusts in a semi-cold desert. Plant Soil 429, 213-225. doi: 10.1007/s11104-017-3300-3

Antoninka, A. J., Bowker, M. A., Reed, S. C., and Doherty, K. (2015). Production of greenhouse-grown biocrust mosses and associated cyanobacteria to rehabilitate dryland soil function. Restor. Ecol. 24, 324-335. doi: 10.1111/rec.12311

Baran, R., Brodie, E. L., Mayberry-Lewis, J., Hummel, E., Da Rocha, U. N., Chakraborty, R., et al. (2015). Exometabolite niche partitioning among sympatric soil bacteria. Nat. Commun. 6:8289. doi: 10.1038/ncomm s9289

Baughman, J., Payton, A. C., Paasch, A. E., Fisher, K. M., and McDaniel, S. F. (2017). Multiple factors influence population sex ratios in the Mojave Desert moss Syntrichia caninervis. Am. J. Bot. 104, 1-10. doi: 10.3732/ajb.1700045

Belnap, J., and Lange, O. L. (2003). Biological Soil Crusts: Structure, Function, and Management. Berlin: Springer. doi: 10.1007/978-3-642-56475-8 
Belnap, J., Phillips, S. L., and Miller, M. E. (2004). Response of desert biological soil crusts to alterations in precipitation frequency. Oecologia 141, 306-316. doi: 10.1007/s00442-003-1438-6

Bolyen, E., Rideout, J. R., Dillon, M. R., Bokulich, N. A., Abnet, C., Al-Ghalith, G. A., et al. (2018). QIIME 2: Reproducible, interactive, scalable, and extensible microbiome data science. PeerJ Prep. 6:e27295v2. doi: $10.7287 /$ peerj.preprints. $27295 \mathrm{v} 2$

Bowker, M. A., Belnap, J., Büdel, B., Sannier, C., Pietrasiak, N., Eldridge, D. J., et al. (2016). "Controls on distribution patterns of biological soil crusts at micro- to global scales," in Biological Soil Crusts: an Organizing Principle in Drylands, eds. B. Weber, B. Büdel, and J. Belnap (Cham: Springer International Publishing), 173-197. doi: 10.1007/978-3-319-30214-0_10

Callahan, B. J., McMurdie, P. J., Rosen, M. J., Han, A. W. Johnson, A. J., and Homes, S. P. (2016). DADA2: high-resolution sample inference from Illumina amplicon data. Nat. Methods 13, 581-583. doi: 10.1038/nmeth.3869

Chan, Y., Lacap, D. C., Lau, M. C. Y., Ha, K. Y., Warren-Rhodes, K. A., Cockell, C. S., et al. (2012). Hypolithic microbial communities: between a rock and a hard place. Environ. Microbiol. 14, 2272-2282. doi: 10.1111/j.1462-2920.2012.02821.x

Cockell, C. S., and Stokes, M. D. (2006). Hypolithic colonization of opaque rocks in the Arctic and Antarctic polar desert. Arct. Antarct. Alp. Res. 38, 335-342. doi: 10.1657/1523-0430(2006)38[335:HCOORI]2.0.CO;2

Couradeau, E., Giraldo-Silva, A., De Martini, F., and Garcia-Pichel, F. (2019). Spatial segregation of the biological soil crust microbiome around its foundational cyanobacterium, Microcoleus vaginatus, and the formation of a nitrogen-fixing cyanosphere. Microbiome 7:55. doi: 10.1186/s40168-019-0661-2

Cowan, D. A., Khan, N., Pointing, S. B., and Cary, S. C. (2010). Diverse hypolithic refuge communities in the McMurdo Dry Valleys. Antarct. Sci. 22, 714-720. doi: 10.1017/S0954102010000507

Cowan, D. A., Sohm, J. A., Makhalanyane, T. P., Capone, D. G., Green, T. G. A., Cary, S. C., et al. (2011). Hypolithic communities: important nitrogen sources in Antarctic desert soils. Env. Microbiol. Rep. 3, 581-586. doi: 10.1111/j.1758-2229.2011.00266.x

Darwin, C. R. (1859). On the Origins of Species by Means of Natural Selection, 6th Edn, Vol. 5. London: John Murray.

de los Ríos, A., Cary, C., and Cowan, D. (2014). The spatial structures of hypolithic communities in the Dry Valleys of East Antarctica. Polar Biol. 37, 1823-1833. doi: 10.1007/s00300-014-1564-0

Delgado-Baquerizo, M., Maestre, F. T., Eldridge, D. J., Bowker, M. A., Ochoa, V., Gozalo, B., et al. (2016). Biocrust-forming mosses mitigate the negative impacts of increasing aridity on ecosystem multifunctionality in drylands. New Phyto. 209, 1540-1552. doi: 10.1111/nph.13688

Durham, R. A., Doherty, K. D., Antoninka, A. J., Ramsey, P. W., and Bowker, M. A. (2018). Insolation ad disturbance history drive biocrust biodiversity in Western Montana rangelands. Plant Soil 430, 151-169. doi: 10.1007/s11104-018-3725-3

Elbert, W., Weber, B., Burrows, S., Steinkamp, J., Büdel, B., Andreae, M. O., et al. (2012). Contribution of cryptogamic covers to the global cycles of carbon and nitrogen. Nat. Geosci. 5, 459-462. doi: 10.1038/ngeo1486

Ettema, C. H., and Wardle, D. A. (2002). Spatial soil ecology. Trends Ecol. Evol. 17, 177-183. doi: 10.1016/S0169-5347(02)02496-5

Garcia-Pichel, F., Loza, V., Marusenko, Y., Mateo, P., and Potrafka, R. M. (2013). Temperature drives the continental-scale distribution of key microbes in topsoil communities. Science 28, 1574-1577. doi: 10.1126/science.1236404

Gause, G. F. (1934). The Struggle for Existence. Baltimore, MD: Williams and Wilkins Company. doi: 10.5962/bhl.title.4489

Giller, K. E., Beare, M. H., Lavelle, P. A., Izac, N., and Swift, M. J. (1997). Agricultural intensification, soil biodiversity and agrosystem function. Agric. Ecosyst. Environ. Appl. Soil Ecol. 6, 3-16. doi: 10.1016/S0929-1393(96)00149-7

Girvan, M. S., Campbell, C. D., Killham, K., Prosser, J. I., and Glover, L. A. (2005). Bacterial diversity promotes community stability and functional resilience after perturbation. Env. Microbiol. 7, 301-313. doi: 10.1111/j.1462-2920.2005.00695.x

Herlemann, D., Labrenz, M., J?rgens, K., Bertilsson, S., Waniek, J. J., and Andersson, A. F. (2011). Transitions in bacterial communities along the $2000 \mathrm{~km}$ salinity gradient of the Baltic Sea. ISME J. 5, 1571-1579. doi: 10.1038/ismej.2011.41

Hutchinson, G. E. (1959). Homage to santa rosalia or why are there so many kinds of animals? Am. Nat. 93, 145-159. doi: 10.1086/282070
Khan, N., Tuffin, M., Stafford, W., Cary, C., Lacap, D. C., Pointing, S. B., et al. (2011). Hypolithic microbial communities of quartz rocks from Miers Valley, McMurdo Dry Valleys, Antarctica. Polar Biol. 34, 1657-1668. doi: 10.1007/s00300-011-1061-7

Kidron, G.J., Barzilay, E., and Sachs, E. (2000). Microclimate control upon microbiotic crusts, western Negev Desert, Israel. Geomorphology 36, 1-18. doi: 10.1016/S0169-555X(00)00043-X

Kidron, G. J., Vonshak, A., and Abeliovich, A. (2009). Microbiotic crusts as biomarkers for surface stability and wetness duration in the Negev Desert. Earth Surf. Proc. Land. 34, 1594-1604. doi: 10.1002/esp.1843

Kidron, G. J., Vonshak, A., Dor, I., Barinova, S., and Abeliovich, A. (2010). Properties and spatial distribution of microbiotic crusts in the Negev Desert, Israel. Catena 82, 92-101. doi: 10.1016/j.catena.2010.05.006

Lacap-Bugler, D. C., Lee, K. K., Archer, S., Gillman, L. N., Lau, M. C. Y., Leuzinger, S., et al. (2017). Global diversity of desert hypolithic Cyanobacteria. Front. Microbiol. 8:867. doi: 10.3389/fmicb.2017.00867

Lange, O. L., Kidron, G. J., Budel, B. A., Meyer, A., Killan, E., and Abeliovich, A. (1992). Taxonomic composition and photosynthetic characteristics of the 'biological soil crusts' covering sand dunes in the western Negev Desert. Funct. Ecol. 6, 519-527. doi: 10.2307/2390048

Le, P. T., Makhalanyane, T. P., Guerrero, L. D., Vikram, S., Van de Peer, Y., and Cowan, D. A. (2016). Comparative metagenomic analysis reveals mechanisms for stress response in hypoliths from extreme hyperarid deserts. Genome Biol. Evol. 8, 2737-2747. doi: 10.1093/gbe/evw189

Lee, K. C., Archer, S. D. J., Boyle, R. H., Lacap-Bugler, D. C., Belnap, J., and Pointing, S. B. (2016). Niche filtering of bacteria in soil and rock habitats of the Colorado Plateau Desert, Utah, USA. Front. Microbiol. 7:1489. doi: 10.3389/fmicb.2016.01489

MacArthur, R. H. (1955). Fluctuations of animal populations and a measure of community stability. Ecology 36, 533-536. doi: 10.2307/1929601

Maier, S., Schmidt, T. S. B., Zheng, L., Peer, T., Wagner, V., and Grube, M. (2014). Analyses of dryland biological soil crusts highlight lichens as an important regulator of microbial communities. Biodivers. Conserv. 23, 1735-1755. doi: 10.1007/s10531-014-0719-1

Maier, S., Tamm, A., Wu, D., Caesar, J., Grube, M., and Weber, B. (2018). Photoautotrophic organisms control microbial abundance, diversity, and physiology in different types of biological soil crusts. ISME J. 12, 1032-1046. doi: 10.1038/s41396-018-0062-8

Makhalanyane, T. P., Valverde, A., Lacap, D. C., Pointing, S. B., Tuffin, M. I., and Cowan, D. A. (2013). Evidence of species recruitment and development of hot desert hypolithic communities. Env. Microbiol. Rep. 5, 219-224. doi: $10.1111 / 1758-2229.12003$

Marquardt, J., and Palinska, K. A. (2007). Genotypic and phenotypic diversity of cyanobacteria assigned to the genus Phormidium (Oscillatoriales) from different habitats and geographical sites. Arch. Microbiol. 187, 397-413. doi: 10.1007/s00203-006-0204-7

McMurdie, P. J., and Holmes, S. (2013). phyloseq: an R package for reproducible interactive analysis and graphics of microbiome census data. PLoS ONE 8:e61217. doi: 10.1371/journal.pone.0061217

Mizrahi-Man, O., Davenport, E. R., and Gilad, Y. (2013). Taxonomic classification of bacterial 16S rRNA genes using short sequencing reads: evaluation of effective study designs. PLoS ONE 8:e53608. doi: 10.1371/journal.pone.0053608

Mogul, R., Vaishampayan, P., Bashir, M., McKay, C. P., Schubert, K., Bornaccorsi, R., et al. (2017). Microbial community and biochemical dynamics of biological soil crusts across a gradient of surface coverage in the central Mojave Desert. Front. Microbiol. 8:1974. doi: 10.3389/fmicb.2017.01974

Moquin, S. A., Garcia, J. R., Brantley, S. L., Takacs-Vesbach, C. D., and Shepherd, U. L. (2012). Bacterial diversity of bryophyte-dominant biological soil crusts and associated mites. J. Arid Environ. 87, 110-117. doi: 10.1016/j.jaridenv.2012.05.004

Pedregosa, F., Varoquaux, G., Gramfort, A., Michel, V., Thirion, B., Grisel, O., et al. (2011). Scikit-learn: machine learning in Python. J. Mach. Learn. Res. 12, 2825-2830. Available online at: http://www.jmlr.org/papers/volume12/ pedregosa11a/pedregosa11a.pdf

Pointing, S. B. (2016). "Hypolithic Communities," in Biological Soil Crusts: an Organizing Principle in Drylands, eds B. Weber, B. Büdel, and J. Belnap (Cham: Springer International Publishing), 199-213. doi: 10.1007/978-3-319-30214-0_11 
Pointing, S. B., and Belnap, J. (2012). Microbial colonization and controls in dryland systems. Nat. Rev. Microbiol. 10:654. doi: 10.1038/nrmicro2854

Quast, C., Pruesse, E., Yilmaz, P., Gerken, J., Schweer, T., Yarza, P., et al. (2013). The SILVA ribosomal RNA gene database project: improved data processing and web-based tools. Nucl. Acids Res. 41, D590-D596. doi: 10.1093/nar/ gks1219

Rosenzweig, M. L. (1981). A theory of habitat selection. Ecology 62, 327-335. doi: $10.2307 / 1936707$

Stomeo, F., Valverde, A., Pointing, S. B., McKay, C. P., Warren-Rhodes, K. A., Tuffin, M. I., et al. (2013). Hypolithic and soil microbial community assembly along an aridity gradient in the Namib Desert. Extremophiles 17, 329-337. doi: 10.1007/s00792-013-0519-7

Suda, S., Watanabe, M. M., Otsuka, S., Mahakahant, A., Yongmanitchai, W., Nopartnaraporn, N., et al. (2002). Taxonomic revision of water-bloomforming species of oscillatorioid cyanobacteria. Int. J. Syst. Evol. Microbiol. 52, 1577-1595. doi: 10.1099/00207713-52-5-1577

Van Goethem, M. W., Makhalanyane, T. P., Cowan, D. A., and Valverde, A. (2017). Cyanobacteria and Alphaproteobacteria may facilitate cooperative interactions in niche communities. Front. Microbiol. 8:2099. doi: 10.3389/fmicb.2017.02099

Vikram, S., Guerrero, L. D., Makhalanyane, T. P., Le, P. T., Seely, M., and Cowan, D. A. (2016). Functional capacity in Namib Hypoliths. Environ. Microbiol. 18, 1875-1888. doi: 10.1111/1462-2920.13088

Weber, B., Bowker, M., Zhang, Y., and Belnap, J. (2016). "Natural recovery of biological soil crusts after disturbance," in Biological Soil Crusts: an Organizing Principle in Drylands, eds B. Weber, B. Büdel, and J. Belnap (Cham: Springer International Publishing), 479-98. doi: 10.1007/978-3-319-30214-0_23
Xiao, B., Hu, K., Ren, T., and Li, B. (2016). Moss-dominated biological soil crusts significantly influence soil moisture and temperature regimes in semiarid ecosystems. Geoderma 263, 35-46. doi: 10.1016/j.geoderma.2015.09.012

Xiao, B., and Veste, M. (2017). Moss-dominated biocrusts increase soil microbial abundance and community diversity and improve soil fertility in semi-arid climates on the Loess Plateau of China. Appl. Soil Ecol. 117-118, 165-177. doi: 10.1016/j.apsoil.2017.05.005

Yilmaz, P., Parfrey, L. W., Yarza, P., Gerken, J., Pruesse, E., Quast, C., et al. (2014). The SILVA and "All-species Living Tree Project (LTP)" taxonomic frameworks. Nucl. Acids Res. 42, D643-D648 doi: 10.1093/nar/gkt1209

Zakrzewski, M., Proietti, C., Ellis, J. J., Hasan, S., Brion, M. J., Berger, B., et al. (2017). Calypso: a user-friendly web-server for mining and visualizing microbiome-environment interactions. Bioinformatics 33, 782-783. doi: 10.1093/bioinformatics/btw725

Conflict of Interest: The authors declare that the research was conducted in the absence of any commercial or financial relationships that could be construed as a potential conflict of interest.

Copyright (C) 2020 Fisher, Jefferson and Vaishampayan. This is an open-access article distributed under the terms of the Creative Commons Attribution License (CC BY). The use, distribution or reproduction in other forums is permitted, provided the original author(s) and the copyright owner(s) are credited and that the original publication in this journal is cited, in accordance with accepted academic practice. No use, distribution or reproduction is permitted which does not comply with these terms. 